\title{
A QUADRATIC PROGRAMMING MODEL FOR OBTAINING WEAK FUZZY SOLUTION TO FUZZY LINEAR SYSTEMS
}

\author{
Abbas Akrami and Majid Erfanian
}

(C) 2019 by University of Niš, Serbia | Creative Commons Licence: CC BY-NC-ND

\begin{abstract}
Real life applications arising in various fields of engineering and science (e.g. electrical, civil, economics, dietary, etc.) can be modelled using a system of linear equations. In such models, it may happen that the values of the parameters are not known or they cannot be stated precisely and that only their estimation due to experimental data or experts knowledge is available. In such a situation it is convenient to represent such parameters by fuzzy numbers. In this paper we propose an efficient optimization model for obtaining a weak fuzzy solution to fuzzy linear systems (FLS). We solve some examples and we show that this method is always efficient.
\end{abstract}

Keywords. Experimental data; fuzzy numbers; fuzzy solution; fuzzy linear systems.

\section{Introduction}

Fuzzy numbers are one way to describe the vagueness and lack of precision of data. The concept of fuzzy numbers and arithmetic operations with these numbers were first introduced and investigated by Zadeh [4] and [13] Mizumoto and Tanaka [9] and [10], Dubois and Prade [7] and Nahmias [11]. One of the major applications using fuzzy number arithmetic is treating linear systems whose parameters all are or partially represented by fuzzy numbers and called fuzzy linear systems (FLS). Many authors have investigated the solution to fuzzy linear systems( [1], [2], [6] and [8]) and all of them make use of the definition given in [8] for converting non-fuzzy solutions to weak fuzzy solutions. In 1998, Friedman et al. [8] proposed a general method for obtaining a solution of a $n \times n$ FLS, whose coefficient matrix is crisp and the right-hand side column is an arbitrary fuzzy number vector. They used the embedding method given in [5] and replaced the original $n \times n$ FLS by a $2 n \times 2 n$ crisp linear system (CLS). The new obtained system was solved and the solution vector was called either a strong fuzzy solution or a weak fuzzy solution to the original fuzzy system.

Received August 15, 2018; Accepted December 14, 2018

2010 Mathematics Subject Classification. Primary 03B52; Secondary 90C20, 94D05 
Hitherto, many researchers have used Friedman et al.'s method. To solve $2 n \times 2 n$ CLS various methods have been employed along with and the mentioned definition in [8]. All researches took it for granted that the weak fuzzy solution defined by Friedman et al. is always a fuzzy number vector, i.e. all of the vector's components are fuzzy numbers.

Afterwards, T.Allahviranloo et al. [3] showed in an example that Friedman et al.'s weak solution may not be a fuzzy vector.

In this paper, we proposed a new method which guarantees that a weak fuzzy solution obtained by this method is always a fuzzy number vector.

\section{Fuzzy Linear Systems}

We represent an arbitrary fuzzy number as follows.

Definition 2.1. [3] The parametric form of an arbitrary fuzzy number $\tilde{a}$ is presented by an ordered pair of functions $(\underline{a}(r), \bar{a}(r)), 0 \leq r \leq 1$, which satisfy the following requirements:

- $\underline{a}(r)$ is a bounded left-continuous non-decreasing function over $[0,1]$.

- $\bar{a}(r)$ is a bounded left-continuous non-increasing function over $[0,1]$.

- $\underline{a}(1)=\bar{a}(1)$.

- $\underline{a}(r) \leq \bar{a}(r), 0 \leq r \leq 1$.

Remark 2.1. If $t \in(0,1)$ be a fixed number and

$$
\underline{a}(r)=\left\{\begin{array}{l}
\alpha_{1}+\beta_{1} r, \quad 0 \leq r \leq t ; \\
\alpha_{1}^{\prime}+\beta_{1}^{\prime} r, \quad t \leq r \leq 1 ;
\end{array} \quad, \bar{a}(r)= \begin{cases}\alpha_{2}-\beta_{2} r, & 0 \leq r \leq t \\
\alpha_{2}^{\prime}-\beta_{2}^{\prime} r, & t \leq r \leq 1 .\end{cases}\right.
$$

then based on Definition 2.1, $\tilde{a}=(\underline{a}(r), \bar{a}(r))$ is a fuzzy number iff

$$
\begin{aligned}
& \alpha_{1}+\beta_{1} t=\alpha_{1}^{\prime}+\beta_{1}^{\prime} t, \\
& \alpha_{2}-\beta_{2} t=\alpha_{2}^{\prime}-\beta_{2}^{\prime} t, \\
& \alpha_{1}^{\prime}+\beta_{1}^{\prime}=\alpha_{2}^{\prime}-\beta_{2}^{\prime}, \\
& \alpha_{1} \leq \alpha_{2}, \alpha_{1}^{\prime} \leq \alpha_{2}^{\prime}, \\
& \beta_{1}, \beta_{1}^{\prime}, \beta_{2}, \beta_{2}^{\prime} \geq 0 .
\end{aligned}
$$

Example 2.1. [3] According to Definition 2.1, the number $\tilde{a}=(\underline{a}(r), \bar{a}(r))$ where

$$
\underline{a}(r)= \begin{cases}8 r-5, & 0 \leq r \leq \frac{1}{2} \\ 4 r-3, & \frac{1}{2} \leq r \leq 1\end{cases}
$$

and

$$
\bar{a}(r)= \begin{cases}4-4 r, & 0 \leq r \leq \frac{1}{2} \\ 3-2 r, & \frac{1}{2} \leq r \leq 1\end{cases}
$$

is a fuzzy number which satisfies the conditions of the remark 2.1. 
Definition 2.2. [8] The following $n \times n$ linear system is called an FLS:

$$
\begin{aligned}
k_{11} x_{1}+k_{12} x_{2}+\ldots+k_{1 n} x_{n} & =\tilde{b_{1}} \\
k_{21} x_{1}+k_{22} x_{2}+\ldots+k_{2 n} x_{n} & =\tilde{b_{2}}, \\
\vdots & \\
k_{n 1} x_{1}+k_{n 2} x_{2}+\ldots+k_{n n} x_{n} & =\tilde{b_{n}},
\end{aligned}
$$

where the coefficients matrix $A=\left(k_{i j}\right), 1 \leq i, j \leq n$, is a crisp $n \times n$ matrix and $\tilde{b}_{i}, 1 \leq i \leq n$, are fuzzy numbers.

Definition 2.3. [8] A fuzzy number vector $\left(x_{1}, x_{2}, \ldots, x_{n}\right)^{T}$ is given by $x_{i}=$ $\left(\underline{x}_{i}(r), \bar{x}_{i}(r)\right), 1 \leq i \leq n, 0 \leq r \leq 1$, is called a solution of the FLS (2.2) if

$$
\underline{\sum_{j=1}^{n} k_{i j} x_{j}}=\sum_{j=1}^{n} \underline{k_{i j} x_{j}}=\underline{b_{i}}, \overline{\sum_{j=1}^{n} k_{i j} x_{j}}=\sum_{j=1}^{n} \overline{k_{i j} x_{j}}=\overline{b_{i}}
$$

according to the proposed model by Friedman et al. we convert the $n \times n$ FLS $(2.2)$ to the following $2 n \times 2 n$ CLS:

$$
T X=B
$$

where

$$
X=\left(\underline{x_{1}}, \ldots, \underline{x_{n}},-\overline{x_{1}}, \ldots,-\overline{x_{n}}\right)^{t}, B=\left(\underline{b_{1}}, \ldots, \underline{b_{n}},-\overline{b_{1}}, \ldots,-\overline{b_{n}}\right)^{t},
$$

and $T_{i j}$ determined as follows:

$$
\begin{aligned}
& k_{i j} \geq 0 \Rightarrow T_{i j}=k_{i j}, T_{i+n, j+n}=k_{i j}, \\
& k_{i j}<0 \Rightarrow T_{i, j+n}=-k_{i j}, T_{i+n, j}=-k_{i j},
\end{aligned}
$$

and any $T_{i j}$ which is not determined by the above equations is zero. Having calculated $X$ which solves Eq.(2.4) and on the assumption $T$ is nonsingular, Friedman et al. defined the "fuzzy solution" to the original system given by Eqs. (2.2) as below.

Definition 2.4. [8] Let the unique solution to CLS (2.4) be denoted by:

$$
X=\left\{\left(\underline{x}_{i}(r),-\bar{x}_{i}(r)\right), 1 \leq i \leq n\right\},
$$

then the fuzzy number vector $W=\left\{\left(\underline{w}_{i}(r), \bar{w}_{i}(r)\right), 1 \leq i \leq n\right\}$ defined by

$$
\begin{aligned}
& \underline{w}_{i}(r)=\min \left\{\underline{x}_{i}(r), \bar{x}_{i}(r), \underline{x}_{i}(1), \bar{x}_{i}(1)\right\}, \\
& \bar{w}_{i}(r)=\max \left\{\underline{x}_{i}(r), \bar{x}_{i}(r), \underline{x}_{i}(1), \bar{x}_{i}(1)\right\},
\end{aligned}
$$

is called the fuzzy solution of $(2.4)$.

If $\left(\underline{x}_{i}(r), \bar{x}_{i}(r)\right), 1 \leq i \leq n, 0 \leq r \leq 1$, are all fuzzy numbers then $\underline{w}_{i}(r)=$ $\underline{x}_{i}(r), \bar{w}_{i}(r)=\bar{x}_{i}(r), 1 \leq i \leq n$, and $W$ is called a strong fuzzy solution. Otherwise, $W$ is called a weak fuzzy solution. 
It should be noted that replacing $\bar{x}_{i}$ and $\underline{x}_{i}$ by $\bar{w}_{i}$ and $\underline{w}_{i}$ does not give the exact equality in (2.3) that is, a weak solution is not a solution to (2.2). Therefore, a weak solution does not satisfy the original problem (2.2). Based on Definition 2.4, Friedman et al. claimed that their weak solution always produces a fuzzy number vector.

T.Allahviranloo et al. in [3] gave an example that this claim is not always true.

Example 2.2. [3] consider the following FLS:

$$
\left\{\begin{array}{c}
x_{1}+x_{2}=\tilde{b_{1}}, \\
x_{1}+2 x_{2}=\tilde{b_{2}},
\end{array}\right.
$$

where

$$
\tilde{b_{1}}=\left(\underline{b_{1}}(r), \overline{b_{1}}(r)\right), \quad \tilde{b_{2}}=\left(\underline{b_{2}}(r), \overline{b_{2}}(r)\right),
$$

and

$$
\begin{gathered}
\underline{b_{1}}(r)=\left\{\begin{array}{cc}
8 r-14, & 0 \leq r \leq \frac{1}{2}, \\
2 r-11, & \frac{1}{2} \leq r \leq 1,
\end{array}, \overline{b_{1}}(r)= \begin{cases}-1-13 r, & 0 \leq r \leq \frac{1}{2}, \\
-6-3 r, & \frac{1}{2} \leq r \leq 1,\end{cases} \right. \\
\underline{b_{2}}(r)=\left\{\begin{array}{cc}
12 r-24, & 0 \leq r \leq \frac{1}{2}, \\
6 r-21, & \frac{1}{2} \leq r \leq 1,
\end{array}, \overline{b_{2}}(r)= \begin{cases}-2-18 r, & 0 \leq r \leq \frac{1}{2}, \\
-7-8 r, & \frac{1}{2} \leq r \leq 1 .\end{cases} \right.
\end{gathered}
$$

The authors in [3] solved this system and obtained the following solution:

$$
\underline{x_{1}}(r)=\left\{\begin{array}{ll}
4 r-4, & 0 \leq r \leq \frac{1}{2}, \\
-2 r-1, & \frac{1}{2} \leq r \leq 1,
\end{array}, \overline{x_{1}}(r)= \begin{cases}-8 r, & 0 \leq r \leq \frac{1}{2} \\
2 r-5, & \frac{1}{2} \leq r \leq 1\end{cases}\right.
$$

and $\underline{x_{2}}(r)=4 r-10, \overline{x_{2}}(r)=-1-5 r$.

Obviously, $x_{2}$ is a fuzzy number and $x_{1}$ is not a fuzzy number(see figure 3.1). By use of Definition 2.4 , the vector $W=\left(\tilde{w}_{1}, \tilde{w}_{2}\right)$ must be a fuzzy number, but we have

$$
\underline{w}_{1}(r)=\left\{\begin{array}{ll}
4 r-4, & 0 \leq r \leq \frac{1}{4}, \\
-3, & \frac{1}{4} \leq r \leq \frac{3}{8}, \\
-8 r, & \frac{3}{8} \leq r \leq \frac{1}{2}, \\
2 r-5, & \frac{1}{2} \leq r \leq 1,
\end{array} \quad, \quad \overline{w_{1}}(r)= \begin{cases}-8 r, & 0 \leq r \leq \frac{1}{3}, \\
4 r-4, & \frac{1}{3} \leq r \leq \frac{1}{2}, \\
-2 r-1, & \frac{1}{2} \leq r \leq 1,\end{cases}\right.
$$

and $\underline{w_{2}}(r)=4 r-10, \overline{w_{2}}(r)=-1-5 r$.

It is clear that $\tilde{w}_{2}=\left(\underline{w}_{2}, \bar{w}_{2}\right)$ is a fuzzy number, whereas $\tilde{w}_{1}=\left(\underline{w}_{1}, \bar{w}_{1}\right)$ is not a fuzzy number. In fact $\underline{w_{1}}(r)$ and $\overline{w_{1}}(r)$ are not non-decreasing and non-increasing functions over $\left[\frac{3}{8}, \frac{1}{2}\right]$ and $\left[\frac{1}{3}, \frac{1}{2}\right]$, respectively. In the next section we propose an optimization model that can be applied for approximation solution to FLS (2.2) which is always a fuzzy number vector. Typically, we solve the example 2.2 and show that the solution which is obtained by this method is a fuzzy number vector. 


\section{Proposed Model}

In this section, we show that the approximation solution of FLS (2.2) with this method becomes a quadratic programming(QP) problem.

A general form of QP problem is as follows:

$$
\begin{array}{ll}
\min & q(x)=\frac{1}{2} x^{T} G x+x^{T} c \\
\text { s.t. } & \\
& A x \leq b,
\end{array}
$$

where $G$ is a symmetric $n \times n$ matrix and $A$ is the $m \times n$ matrix, jacobian of constraints, and $b$ is a vector in $\operatorname{Re}^{m}$. We solve the problem (3.1) by use of the active set method [12].

Definition 3.1. Let $v=(\underline{v}(r), \bar{v}(r))$ where

$$
\underline{v}(r)=\left\{\begin{array}{ll}
a_{1}+b_{1} r, & 0 \leq r \leq t, \\
a_{1}^{\prime}+b_{1}^{\prime} r, & t \leq r \leq 1,
\end{array}, \bar{v}(r)= \begin{cases}a_{2}-b_{2} r, & 0 \leq r \leq t, \\
a_{2}^{\prime}-b_{2}^{\prime} r, & t \leq r \leq 1,\end{cases}\right.
$$

which is not satisfied with the definition 2.1, i.e. $v$ is not a fuzzy number and we want to approximate it with a fuzzy number $u=(\underline{u}(r), \bar{u}(r))$, where

$$
\underline{u}(r)=\left\{\begin{array}{c}
\alpha_{1}+\beta_{1} r, \quad 0 \leq r \leq t, \\
\alpha_{1}^{\prime}+\beta_{1}^{\prime} r, \quad t \leq r \leq 1,
\end{array}, \bar{u}(r)=\left\{\begin{array}{cc}
\alpha_{2}-\beta_{2} r, & 0 \leq r \leq t, \\
\alpha_{2}^{\prime}-\beta_{2}^{\prime} r, & t \leq r \leq 1
\end{array}\right.\right.
$$

We say that $u$ is an approximation for $v$ iff it is a solution of the following optimization problem:

$$
\begin{array}{cl}
\min & \|p-q\|^{2} \\
\text { s.t. } & \\
& \alpha_{1}+\beta_{1} t=\alpha_{1}^{\prime}+\beta_{1}^{\prime} t, \\
& \alpha_{2}-\beta_{2} t=\alpha_{2}^{\prime}-\beta_{2}^{\prime} t, \\
& \alpha_{1}^{\prime}+\beta_{1}^{\prime}=\alpha_{2}^{\prime}-\beta_{2}^{\prime}, \\
& \beta_{1}, \beta_{1}^{\prime}, \beta_{2}, \beta_{2}^{\prime} \geq 0, \\
& \alpha_{1} \leq \alpha_{2}, \\
& \alpha_{1} \leq \alpha_{2},
\end{array}
$$

where $p=\left(\alpha_{1}, \beta_{1}, \alpha_{1}^{\prime}, \beta_{1}^{\prime}, \alpha_{2}, \beta_{2}, \alpha_{2}^{\prime}, \beta_{2}^{\prime}\right), q=\left(a_{1}, b_{1}, a_{1}^{\prime}, b_{1}^{\prime}, a_{2}, b_{2}, a_{2}^{\prime}, b_{2}^{\prime}\right)$ are corresponding vectors with $v$ and $u$ respectively.

Remark 3.1. If we rewrite the problem (3.2) into a matrix form then we have:

$$
\begin{array}{cl}
\min & f(x)=\frac{1}{2} x^{T} G x+x^{T} c \\
\text { s.t. } & \\
& A x \leq b,
\end{array}
$$


where $G=2 I_{8 \times 8}$ (I is identity matrix), $c=-2\left[a_{1}, b_{1}, a_{1}^{\prime}, b_{1}^{\prime}, a_{2}, b_{2}, a_{2}^{\prime}, b_{2}^{\prime}\right]^{t}$ and

$$
A=\left(\begin{array}{cccccccc}
0 & 0 & 1 & 1 & 0 & 0 & -1 & 1 \\
0 & 0 & -1 & -1 & 0 & 0 & 1 & -1 \\
1 & t & -1 & -t & 0 & 0 & 0 & 0 \\
-1 & -t & 1 & t & 0 & 0 & 0 & 0 \\
0 & 0 & 0 & 0 & -1 & t & 1 & -t \\
0 & -1 & 0 & 0 & 0 & 0 & 0 & 0 \\
0 & 0 & 0 & -1 & 0 & 0 & 0 & 0 \\
0 & 0 & 0 & 0 & 0 & -1 & 0 & 0 \\
1 & 0 & 0 & 0 & -1 & 0 & 0 & 0 \\
0 & 0 & 1 & 0 & 0 & 0 & -1 & 0
\end{array}\right) .
$$

Now, using the model (3.3) for the $x_{2}$ in the example 2.2 and solving it with the active-set method, the weak fuzzy solution $z=\left(z_{1}, z_{2}\right)$ is obtained as follows:

$$
\underline{z_{1}}(r)=\left\{\begin{array}{ll}
-4.8+3.6 r, & 0 \leq r \leq \frac{1}{2}, \\
-3, & \frac{1}{2} \leq r \leq 1,
\end{array}, \quad \overline{z_{1}}(r)= \begin{cases}0.8-7.6 r, & 0 \leq r \leq \frac{1}{2}, \\
-3, & \frac{1}{2} \leq r \leq 1,\end{cases}\right.
$$

and

$$
z_{2}=(4 r-10,-1-5 r) .
$$

By substituting $z$ in the example 2.2 we have:

$$
\left\{\begin{array}{c}
z_{1}+z_{2}=\tilde{y}_{1}, \\
z_{1}+2 z_{2}=\tilde{y}_{2},
\end{array}\right.
$$

where

$$
\tilde{y}_{1}=\left(\underline{y_{1}}(r), \overline{y_{1}}(r)\right), \quad \tilde{y}_{2}=\left(\underline{y_{2}}(r), \overline{y_{2}}(r)\right)
$$

and

$$
\begin{aligned}
& \overline{y_{1}}(r)= \begin{cases}-12.6 r-0.2, & 0 \leq r \leq \frac{1}{2}, \\
-5 r-4, & \frac{1}{2} \leq r \leq 1,\end{cases} \\
& \underline{y_{1}}(r)= \begin{cases}7.6 r-14.8, & 0 \leq r \leq \frac{1}{2}, \\
4 r-13, & \frac{1}{2} \leq r \leq 1 .\end{cases} \\
& \overline{y_{2}}(r)= \begin{cases}-17.6 r-1.2, & 0 \leq r \leq \frac{1}{2}, \\
-10 r-5, & \frac{1}{2} \leq r \leq 1,\end{cases} \\
& \underline{y_{2}}(r)= \begin{cases}11.6 r-24.8, & 0 \leq r \leq \frac{1}{2}, \\
8 r-23, & \frac{1}{2} \leq r \leq 1 .\end{cases}
\end{aligned}
$$

We can see $x_{1}$ and its fuzzy approximation, $z_{1}$, in Figure 3.2

Also, Figure 3.3 and Figure 3.4 show an error in the system. 


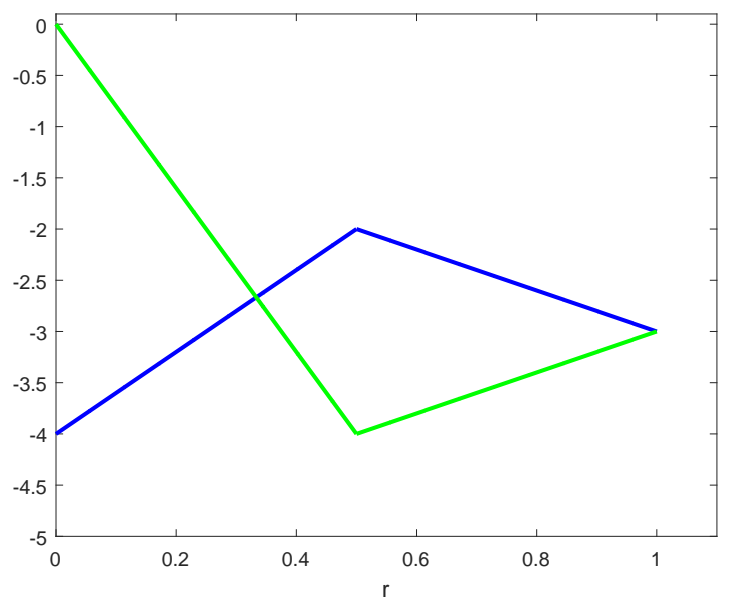

FIG. 3.1: nonfuzzy number $x_{1}$

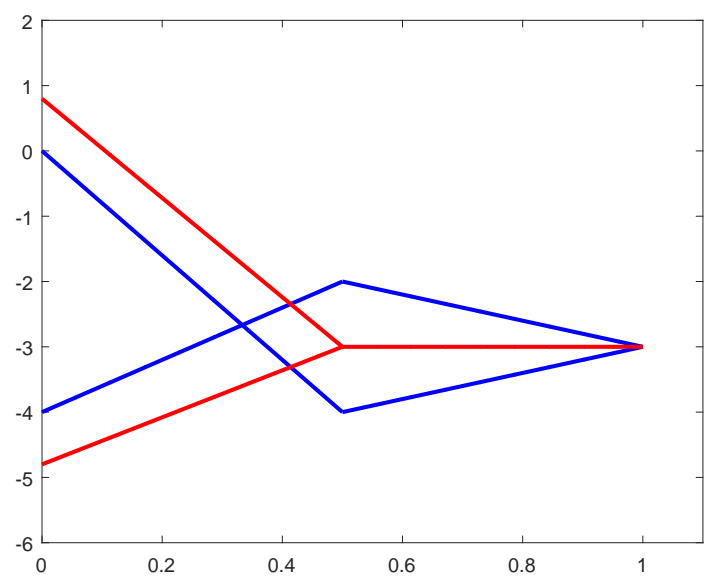

FIG. 3.2: The fuzzy number $z_{1}$ and nonfuzzy number $x_{1}$ 


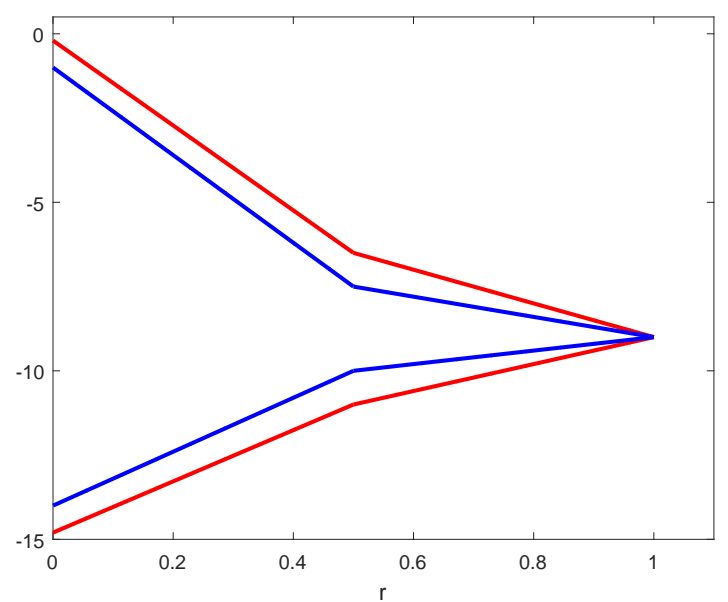

FIG. 3.3: comparing $\tilde{y}_{1}$ and $\tilde{b}_{1}$

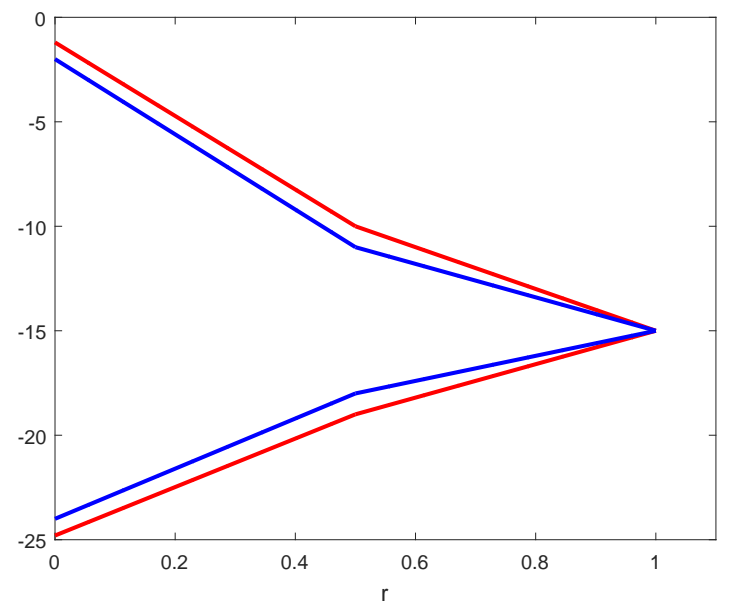

FIG. 3.4: comparing $\tilde{y}_{2}$ and $\tilde{b}_{2}$ 


\section{Conclusion}

In this paper, a new method was presented for obtaining the weak fuzzy solution to fuzzy linear systems. This method can be generalized for any number that is not fuzzy and we can approximate it with the fuzzy number by solving a quadratic programming problem.

\section{REF ER E N C ES}

1. S. Abbasbandy and M. Alavi: A method for solving fuzzy linear systems. Iranian J. of Fuzzy Sys. 2 (2005), 37-43.

2. T. Allahviranloo: Numerical methods for fuzzy system of linear equations. Applied Math. and Computation. 155 (2004), 493-502.

3. T. Allahviranloo and M. Ghanbari and A. A. Hosseinzadeh and E. Haghi and R. Nurae: A note on " Fuzzy linear systems. Fuzzy Sets and Sys. 177 (2011), 87-92.

4. S. S. L. Chang and L. A. ZADeh: On fuzzy mapping and control. IEEE Trqns. Systems Man Cybernet. 2 (1972), 30-34.

5. W. Cong-Xin and M. Ming: Embedding problem of fuzzy number space: Part I. Fuzzy Sets and Sys. 44 (1991), 33-38.

6. M. Dehghan and B. HAShemi: Iterative solution of fuzzy linear systems. Applied Math. and Computation. 175 (2006), 645-674.

7. D. Dubios and H. Prade: Operations on fuzzy numbers. J.Systems Sci. 9 (1978), 13-26.

8. M. Friedman and M. Ming and A. KAndel: Fuzzy linear systems, Fuzzy Sets and Sys. 96 (1998), 201-209.

9. M. Mizumoto and K. TAnaka: The four operations of arithmetic on fuzzy numbers. Systems Comput. Controls. 7 (1976), 73-81.

10. M. Mizumoto and K. Tanaka: Some properties of fuzzy numbers. Asvances in fuzzy set theory and Applications. (1979), 156-164.

11. S. Nahmias: Fuzzy variables. Fuzzy Set and Sys. 2 (1978), 97-111.

12. J. Nocedal and S. J. Wright: Numerical Optimization (2nd ed.). Berlin, New York: Springer-Verlag, 2006.

13. L. A. ZADEH: The concept of a linguistic variable and its application to approximate reasoning. Inform. Sci. 8 (1975), 199-249.

Abbas Akrami(Corresponding Author)

Faculty of Science

Department of Science,School of Mathematical science

University of Zabol, Zabol, Iran

akrami.ab@uoz.ac.ir 
Majid Erfanian

Faculty of Science

Department of Science,School of Mathematical science

University of Zabol, Zabol, Iran

erfaniayn@uoz.ac.ir 